\title{
The influence of stray DC magnetic fields in MeV Ion Nanobeam Systems
}

\author{
M.J. Merchant ${ }^{\mathrm{a}, *}$, G.W. Grime ${ }^{\mathrm{a}}$ \\ ${ }^{a}$ Ion Beam Centre, University of Surrey, Guildford, UK, GU2 7XH
}

\begin{abstract}
The influence of stray AC magnetic fields on the resolution of focussed $\mathrm{MeV}$ ion microbeam and nanobeam systems is well understood, and reduction of such stray fields is critical to achieving sub-micron resolution. However, the influence of stray DC magnetic fields is not so well understood, and impossible to avoid, with the earth providing such a "stray" field over the entire length of the beamline. It is widely assumed that the influence of such fields is negligible when the optical elements of the microbeam system are aligned to the "true" path of the beam through any stray DC fields. In this paper numerical raytracing has been used to study the influence of stray DC magnetic fields on beam resolution at the sub-micron level using typical field strengths for the earth's magnetic field as a case study.

Key words: Nuclear microbeams, Beam optics

PACS: ,
\end{abstract}

\section{Contents}

1 Introduction

2 The magnetic field of the earth 2

3 Simulation 3

3.1 Aberration due to imperfect alignment techniques . . . . . . . . 4

3.2 Increased Chromatic Aberration . . . . . . . . . . . . . 5

3.3 Focussing performance degradation when changing beam energy . . . 10

${ }^{*}$ Corresponding author 


\begin{tabular}{|c|c|c|c|c|c|}
\hline \multicolumn{2}{|c|}{ Lat: $5128^{\prime} 48^{\prime \prime}$} & \multicolumn{2}{|c|}{ Lon: - 10' 12"' } & \multicolumn{2}{|c|}{ Elev: $0.00 \mathrm{~m}$} \\
\hline Declination & Inclination & & & & \\
\hline-154 & $6628^{\prime}$ & & & & \\
\hline Horizontal Intensity & $\begin{array}{l}\text { North Component } \\
+ \text { + North - South }\end{array}$ & $\begin{array}{l}\text { East Component } \\
+ \text { +East - West }\end{array}$ & $\begin{array}{l}\text { Vertical Component } \\
+ \text { Down - Up }\end{array}$ & $\begin{array}{l}\text { Total Field } \\
\end{array}$ & \\
\hline & $19,394.4 \mathrm{nT}$ & $19,383.7 \mathrm{nT}$ & $-643.5 \mathrm{nT}$ & $44,538.7 \mathrm{nT}$ & $48,578.2 \mathrm{nT}$ \\
\hline
\end{tabular}

\section{Conclusion}

\section{Introduction}

Stray DC magnetic fields are widely considered as inconsequential in the design of focussed microbeam systems. Whether consequential or inconsequential, they are unavoidable. The earth generates a magnetic field in the order of 50,000 nanotesla, and with common microbeam lengths greater than 5 metres this results in a deflection in the order of millimetres. The established experimental technique, as described by Grime and Watt[1], is to align the optical elements of the focused system to the true path of the ions through any stray fields, thus ensuring that the ions pass through the optical center of each elements. With this technique, beam deflection resulting from unknown stray DC fields is not noticeable or important to the microbeam user.

It is a good practice to move all electronic equipment as far as reasonably possible from the beamline to reduce the effect of stray magnetic fields, however this is largely related to the essential need to reduce the influence of stray AC magnetic fields which critically alter the resolution of microbeam systems by deflecting the beam about a central point thus bluring the focus. The influence of such stray fields is dependant upon the location of the stray field with respect to the object aperture of the microbeam, since the greater the distance to the image plane, the longer the lever arm of any deflection.

\section{The magnetic field of the earth}

The National Geophysical Data Centre, U.S.A[2] provides values for the strength of the Earth's magnetic field in London, UK. These values are the closest available to the University of Surrey Ion Beam Centre, and as such should be a good approximations to field strengths in Surrey, UK. 
The microbeam system used for simulation in this paper uses three magnetic quadrupole lenses in an Oxford Triplet geometry [4], of 6.367 metres in total length from object to image planes.

For this beamline, the unfocussed path of a $2.5 \mathrm{MeV}$ beam, orientated directly from east to west, will recieve deflections of $-3.96 \mathrm{~mm}$ and $1.7 \mathrm{~mm}$ in the $\mathrm{x}-\mathrm{z}$ and $\mathrm{y}-\mathrm{z}$ axis respectively due to the Earth's magnetic field.

To model the earths magnetic field, a magnetic dipole with a pole tip field of 48,578 nT has been inserted into the simulation of the Oxford Triplet, and rotated by 1.6 radians about the yaxis, and 0.41 radians about the $\mathrm{x}$ axis giving field strengths equal to those shown in table 2 .

This paper studies three concerns arising from the influence of Stray DC magnetic fields on a beamline.

- The first concern involves an evaluation of the alignment procedure used to align relatively thick optical elements to the curved path taken by the beam in the presence of stray DC magnetic fields.

- The second concern is whether chromatic aberration increases due to the changing path of a chromatically spread beam in a DC magnetic field.

- The third concern is the degradation of the focussing power of the microprobe when ions of a different magnetic rigidity are used to that for which the optical elements of the beamline were aligned to.

The third concern is of particular significance, since beam alignment is an arduos process, which may cause great delay to experiments requiring an change of ion rigidities between experimental runs. This is very relevant to the proton beam writing technique which often requires a range of ion rigidities to make multi-level structures.

\section{Simulation}

Simulation of the influence of stray DC magnetic fields using numerical raytracing is achieved by replicating experimental practice for aligning the centre of optical elements to the new beam axis. This can be achieved in the following steps. 
1. Excitation of all active elements in the simulation are set to zero.

2. A magnetic dipole representing the stray field is added to the simulation, and excited.

3. A para-axial ray is traced, and the ray coordinates are recorded of as the ray passes through the centre of each optical element, including object and collimator apertures, and the final position at the image plance is recorded.

4. The pre-object path of the particle is adjusted such that the particle passes through both object and collimating apertures despite the influence of the stay field.

5. The center and tilt of all optical elements in the simulation are adjusted to the recorded positions of the paraxial ray.

6. The excitations of active elements in the simulation are optimised to give a focus at the new image position.

\subsection{Aberration due to imperfect alignment techniques}

The procedure outlined above differs to experimental practice in that the tilt alignment of the lenses has not be corrected to the new beam axis In practice, the tilt misalignment would cause an offset in the line foci, which the user would attempt to correct by translation. The result is that each optical element is misaligned in both translation and tilt.

This aberration arise from the tilt misalignment of the optical elements from the new beam-axis, and may be improved if each optical element is correctly tilted by it's unique amount matching the deviation of the beam-axis as it crosses through the centre of each element. Experimentally, this will be almost impossible to achieve, since translation misalignment and tilt misalignment produce a similar misalignment of the line foci, and it will be impossible for the microbeam operator to distinguish between the two. Perhaps the only way for a microbeam operator to correctly align a microbeam will be using the grid-shadowing technique. Analysis of grid-shadow patterns is complex, and will need to be carried out for for each change in ion rigidity.

The point spread functions shown in figures 1 have been "corrected" in the xplane to show a image position relative the a ray with traced with no input divergence, and thus the input offset of $4.4 \mathrm{~mm}$ has been removed, since this would not be important to 
a microbeam operator. This follows the practice of the microbeam operator, who centre his target viewing aparatus on the beam axis rather than with respect to the object coordinates. However, a $2.5 \mu \mathrm{m}$ misalignment is shown on the point spread functions in the presence of the stray DC field. This is due in part to the steering influence of the imperfectly aligned quadrupoles, and in part to that fact that the image plane is no longer perpendicular to the beam axis, instead it is perpendicular to the coordinate axis. This serves to stretch the point spread function away from the coordinate axis.

The "difference" point spread function shown in figure 3.2 is generated by subtracting the aberration coefficients calculated in the unstrayed matrix from those calculated in the translationally aligned matrix, leaving a polynomial of difference terms. This is shown in table 2 .

The degradation in focus quality due to imperfect alignment techniques is characterised by an increase in 2 nd and 4 th order aberration terms, $\langle x| \theta^{2},\langle x| \phi^{2}$, and in the $\mathrm{y}$-axis particulary the cross-coupling terms, $\langle y \mid \theta \phi\rangle,\left\langle y \mid \theta^{3} \phi\right\rangle,\left\langle y \mid \theta \phi^{3}\right\rangle$.

This is similar in some ways to off-axis scanning aberration observed in ref??

The aberration $\langle y| \theta \phi$ is by far the most dominant, from the set of misalignment and chromatic aberrations introduced by the stray field, and an improvement factor of two in the coefficient alone when the lens are correctly tilted to the beam direction is responsible for the large improvement in translation aligned and tilt aligned point spread functions.

It is clear from examination of the tables 2 and 3 that alignment techniques using both translation and tilt of the optical elements makes a factor of two difference in first order chromatic aberration terms $\langle x \mid \theta \delta\rangle$ and $\langle y \mid \phi \delta\rangle$ broadening terms. This improvement is evident in the difference point spread function shown in figure 3.2, where the difference in aberration can be reduced from $100 \mathrm{~nm}$ to $50 \mathrm{~nm}$.

\subsection{Increased Chromatic Aberration}

The chromatic aberration of the beamline is shown to be increased beyond the values given in traditional measurements due to not just overfocussing of the lens elements, but a first order aberration is introduced due to the chromatically differing beams entering the optical elements off-axis. However this effect is demagnified by the 


\begin{tabular}{|c|c|c|c|c|}
\hline & $\mathrm{X}$ & $\mathrm{y}$ & $\theta$ & $\phi$ \\
\hline$\theta$ & $-2.5009 \mathrm{e}-02$ & & $8.2396 \mathrm{e}+01$ & \\
\hline$\phi$ & & $6.3119 \mathrm{e}-01$ & & $-3.1450 \mathrm{e}+01$ \\
\hline$\theta^{2}$ & & & $1.2095 \mathrm{e}-10$ & \\
\hline$\theta \phi$ & $3.0785 \mathrm{e}-10$ & & & \\
\hline$\theta \delta$ & $-7.8838 \mathrm{e}-03$ & & $-7.8692 \mathrm{e}-05$ & \\
\hline$\phi \delta$ & & $1.7184 \mathrm{e}-02$ & & $8.5326 \mathrm{e}-05$ \\
\hline$\theta^{3}$ & $7.2522 \mathrm{e}+02$ & $2.4111 \mathrm{e}-10$ & $4.8320 \mathrm{e}+00$ & \\
\hline$\theta^{2} \phi$ & $2.2372 \mathrm{e}-10$ & $-3.9574 \mathrm{e}+03$ & & $-1.9902 \mathrm{e}+01$ \\
\hline$\theta \phi^{2}$ & $1.5108 \mathrm{e}+03$ & & $1.2998 \mathrm{e}+01$ & \\
\hline$\theta \delta^{2}$ & $4.6637 \mathrm{e}-09$ & & & \\
\hline$\phi^{3}$ & $-8.2985 \mathrm{e}-10$ & $-4.8029 e+03$ & & $-2.3534 \mathrm{e}+01$ \\
\hline$\phi \delta^{2}$ & & $-1.1198 \mathrm{e}-08$ & & \\
\hline$\theta^{3} \phi$ & $-2.6116 \mathrm{e}-08$ & $-4.3003 e-09$ & & $-1.7167 \mathrm{e}-10$ \\
\hline$\theta^{3} \delta$ & $-1.1439 \mathrm{e}-03$ & & $-6.6283 e-06$ & \\
\hline$\theta^{2} \phi^{2}$ & $4.9787 \mathrm{e}-09$ & $2.2211 \mathrm{e}-10$ & $-5.9076 e-09$ & $-1.0092 \mathrm{e}-10$ \\
\hline$\theta^{2} \phi \delta$ & $2.2301 \mathrm{e}-10$ & $4.4294 \mathrm{e}-03$ & & $2.2186 \mathrm{e}-05$ \\
\hline$\theta \phi^{3}$ & $-3.5606 e-08$ & $-3.1065 \mathrm{e}-09$ & $-2.2493 e-10$ & $-1.7341 \mathrm{e}-10$ \\
\hline$\theta \phi^{2} \delta$ & $-1.4584 \mathrm{e}-03$ & & $-1.0171 \mathrm{e}-05$ & \\
\hline$\phi^{3} \delta$ & $3.0269 \mathrm{e}-10$ & $3.8796 \mathrm{e}-03$ & & $1.9386 \mathrm{e}-05$ \\
\hline$\theta^{3} \phi^{2}$ & $2.0256 \mathrm{e}+02$ & $-3.0169 \mathrm{e}-09$ & $1.1734 \mathrm{e}+00$ & $-1.4904 \mathrm{e}-09$ \\
\hline$\theta^{3} \phi \delta$ & $1.1075 \mathrm{e}-09$ & $-5.9599 \mathrm{e}-10$ & & \\
\hline$\theta^{3} \delta^{2}$ & $8.4387 \mathrm{e}-09$ & & & \\
\hline$\theta^{2} \phi^{3}$ & $-3.4160 \mathrm{e}-08$ & $-7.7770 \mathrm{e}+02$ & $-1.2634 \mathrm{e}-09$ & $-3.6954 \mathrm{e}+00$ \\
\hline$\theta^{2} \phi^{2} \delta$ & $-4.3156 e-10$ & $-3.8125 \mathrm{e}-10$ & $2.4222 \mathrm{e}-10$ & \\
\hline$\theta^{2} \phi \delta^{2}$ & & $1.0405 \mathrm{e}-07$ & & $5.1843 \mathrm{e}-10$ \\
\hline$\theta \phi^{3} \delta$ & $-1.2839 \mathrm{e}-09$ & $-1.1633 e-09$ & & \\
\hline$\theta \phi^{2} \delta^{2}$ & $9.9327 \mathrm{e}-09$ & & & \\
\hline \multirow[t]{5}{*}{$\phi^{3} \delta^{2}$} & & $1.0882 \mathrm{e}-07$ & & $5.5057 \mathrm{e}-10$ \\
\hline & Variable & Order & Range & Mean \\
\hline & $\theta$ & 3 & 0.1 & 0 \\
\hline & $\phi$ & 3 & 0.1 & 0 \\
\hline & $\delta$ & 2 & 10 & $2.5 e+06$ \\
\hline
\end{tabular}

Table 1: Matrix of aberration coefficients for Oxford Triplet without stray DC field. 


\begin{tabular}{|c|c|c|c|c|}
\hline \multicolumn{5}{|c|}{ matrıx: units: $\mu \mathrm{m} /$ mrads $/$} \\
\hline & $\mathrm{X}$ & $\mathrm{y}$ & $\theta$ & $\phi$ \\
\hline & $-2.4430 e+00$ & $-6.4365 e-01$ & $-1.7335 \mathrm{e}-02$ & $8.9429 \mathrm{e}-03$ \\
\hline$\theta$ & $7.8903 e-02$ & $-2.3300 \mathrm{e}-02$ & $6.9136 \mathrm{e}-04$ & $-4.0181 e-04$ \\
\hline$\phi$ & $8.8961 \mathrm{e}-03$ & $-1.4646 \mathrm{e}-01$ & $-6.9174 \mathrm{e}-04$ & $-7.2735 \mathrm{e}-04$ \\
\hline$\delta$ & $-4.8013 e-06$ & $-1.5338 \mathrm{e}-05$ & $9.4484 \mathrm{e}-06$ & $1.5326 \mathrm{e}-06$ \\
\hline$\theta^{2}$ & $2.2359 \mathrm{e}+00$ & $3.2007 \mathrm{e}+00$ & $3.6476 \mathrm{e}-02$ & $1.1944 \mathrm{e}-02$ \\
\hline$\theta \phi$ & $-2.4425 e+00$ & $-2.0535 e+01$ & $-1.1548 \mathrm{e}-02$ & $-1.0031 \mathrm{e}-01$ \\
\hline$\theta \delta$ & $5.7001 \mathrm{e}-07$ & $1.9543 \mathrm{e}-06$ & $8.5953 \mathrm{e}-09$ & $8.7610 \mathrm{e}-09$ \\
\hline$\phi^{2}$ & $3.9294 \mathrm{e}+00$ & $4.2895 \mathrm{e}+00$ & $5.1643 \mathrm{e}-02$ & $1.8295 \mathrm{e}-02$ \\
\hline$\phi \delta$ & $-6.2252 \mathrm{e}-07$ & $-2.6865 e-06$ & $-5.8045 \mathrm{e}-09$ & $-1.2835 \mathrm{e}-08$ \\
\hline$\delta^{2}$ & $-9.1114 \mathrm{e}-10$ & $-8.7519 \mathrm{e}-10$ & & \\
\hline$\theta^{3}$ & $1.3722 \mathrm{e}-02$ & $-3.3749 \mathrm{e}-02$ & $8.0560 \mathrm{e}-05$ & $-1.7046 \mathrm{e}-04$ \\
\hline$\theta^{2} \phi$ & $9.1300 \mathrm{e}-03$ & $-5.0040 \mathrm{e}-02$ & $1.0407 \mathrm{e}-04$ & $-2.4038 \mathrm{e}-04$ \\
\hline$\theta^{2} \delta$ & $2.4948 \mathrm{e}-04$ & $1.9940 \mathrm{e}-04$ & $1.6453 \mathrm{e}-06$ & $1.0056 \mathrm{e}-06$ \\
\hline$\theta \phi^{2}$ & $2.0318 \mathrm{e}-02$ & $-5.6420 \mathrm{e}-02$ & $1.5337 \mathrm{e}-04$ & $-2.7213 e-04$ \\
\hline$\theta \phi \delta$ & $-1.5108 \mathrm{e}-04$ & $-8.9606 \mathrm{e}-04$ & $-1.3083 e-06$ & $-4.5089 \mathrm{e}-06$ \\
\hline$\theta \delta^{2}$ & $6.4473 \mathrm{e}-11$ & $-3.8296 \mathrm{e}-10$ & & \\
\hline$\phi^{3}$ & $-1.2026 \mathrm{e}-02$ & $-6.3311 \mathrm{e}-02$ & $-1.0417 \mathrm{e}-04$ & $-3.0645 e-04$ \\
\hline$\phi^{2} \delta$ & $1.7007 \mathrm{e}-04$ & $7.3383 \mathrm{e}-04$ & $1.4680 \mathrm{e}-06$ & $3.5987 \mathrm{e}-06$ \\
\hline$\phi \delta^{2}$ & & $1.1952 \mathrm{e}-09$ & & \\
\hline$\theta^{3} \phi$ & $-4.1520 \mathrm{e}-01$ & $-2.1834 \mathrm{e}+00$ & $-2.4230 \mathrm{e}-03$ & $-9.2772 \mathrm{e}-03$ \\
\hline$\theta^{3} \delta$ & $1.5488 \mathrm{e}-07$ & $4.9804 \mathrm{e}-07$ & $2.8531 \mathrm{e}-09$ & $2.2300 \mathrm{e}-09$ \\
\hline$\theta^{2} \phi^{2}$ & $1.0334 \mathrm{e}+00$ & $2.4447 \mathrm{e}-01$ & $1.1276 \mathrm{e}-02$ & $2.5752 \mathrm{e}-04$ \\
\hline$\theta^{2} \phi \delta$ & $-2.5222 \mathrm{e}-07$ & $-1.5339 \mathrm{e}-06$ & $-3.3154 \mathrm{e}-09$ & $-7.3185 e-09$ \\
\hline$\theta^{2} \delta^{2}$ & $9.0986 \mathrm{e}-10$ & $5.6077 \mathrm{e}-10$ & & \\
\hline$\theta \phi^{3}$ & $-7.3166 \mathrm{e}-01$ & $-8.6369 e+00$ & $-4.0023 e-03$ & $-4.1786 \mathrm{e}-02$ \\
\hline$\theta \phi^{2} \delta$ & $3.3052 \mathrm{e}-07$ & $1.7821 \mathrm{e}-06$ & $3.9096 \mathrm{e}-09$ & $7.9886 \mathrm{e}-09$ \\
\hline$\theta \phi \delta^{2}$ & $3.2904 \mathrm{e}-09$ & $-3.2060 \mathrm{e}-08$ & & $-1.6130 \mathrm{e}-10$ \\
\hline$\phi^{3} \delta$ & $-2.9875 e-07$ & $-1.8359 \mathrm{e}-06$ & $-2.8092 \mathrm{e}-09$ & $-9.1154 \mathrm{e}-09$ \\
\hline$\phi^{2} \delta^{2}$ & $9.3003 e-11$ & $-2.4559 \mathrm{e}-09$ & & \\
\hline & Variable & Order & Range & Mean \\
\hline & $\theta$ & 3 & 0.1 & 0 \\
\hline & $\phi$ & 3 & 0.1 & 0 \\
\hline & $\delta$ & 2 & 10 & $2.5 e+06$ \\
\hline
\end{tabular}

Table 2: Matrix of aberration coefficients for comparison of beamline with and without stray DC field. 
Aberration matrix: units: $\mu \mathrm{m} / \operatorname{mrads} / \mathrm{eV}$.

\begin{tabular}{|c|c|c|c|c|}
\hline & $\mathrm{X}$ & $\mathrm{y}$ & $\theta$ & $\phi$ \\
\hline & $-1.2093 \mathrm{e}+00$ & $-9.6478 e-01$ & $-8.8377 \mathrm{e}-03$ & $1.3420 \mathrm{e}-02$ \\
\hline$\theta$ & $6.0827 \mathrm{e}-02$ & $-2.2206 \mathrm{e}-02$ & $5.2663 \mathrm{e}-04$ & $-4.2125 e-04$ \\
\hline$\phi$ & $8.4785 \mathrm{e}-03$ & $-1.5715 \mathrm{e}-01$ & $-6.9104 \mathrm{e}-04$ & $-7.7658 \mathrm{e}-04$ \\
\hline$\delta$ & $-6.2457 \mathrm{e}-06$ & $-1.7476 \mathrm{e}-05$ & $9.4316 \mathrm{e}-06$ & $1.5209 \mathrm{e}-06$ \\
\hline$\theta^{2}$ & $1.0671 \mathrm{e}+00$ & $4.7882 e+00$ & $1.8031 \mathrm{e}-02$ & $1.7843 \mathrm{e}-02$ \\
\hline$\theta \phi$ & $-3.6539 e+00$ & $-1.0219 \mathrm{e}+01$ & $-1.6498 \mathrm{e}-02$ & $-4.8341 \mathrm{e}-02$ \\
\hline$\theta \delta$ & $3.7592 \mathrm{e}-07$ & $1.7949 \mathrm{e}-06$ & $4.6691 \mathrm{e}-09$ & $7.4843 \mathrm{e}-09$ \\
\hline$\phi^{2}$ & $1.9554 \mathrm{e}+00$ & $6.3510 \mathrm{e}+00$ & $2.5830 \mathrm{e}-02$ & $2.7034 \mathrm{e}-02$ \\
\hline$\phi \delta$ & $-5.5692 \mathrm{e}-07$ & $-1.7017 \mathrm{e}-06$ & $-3.7322 \mathrm{e}-09$ & $-7.7110 \mathrm{e}-09$ \\
\hline$\delta^{2}$ & $-9.0148 \mathrm{e}-10$ & $-8.6363 e-10$ & & \\
\hline$\theta^{3}$ & $1.0599 \mathrm{e}-02$ & $-3.4864 \mathrm{e}-02$ & $5.7595 \mathrm{e}-05$ & $-1.7669 \mathrm{e}-04$ \\
\hline$\theta^{2} \phi$ & $9.2876 \mathrm{e}-03$ & $-3.4214 \mathrm{e}-02$ & $1.0864 \mathrm{e}-04$ & $-1.6094 \mathrm{e}-04$ \\
\hline$\theta^{2} \delta$ & $2.5019 \mathrm{e}-04$ & $1.9762 \mathrm{e}-04$ & $1.6579 \mathrm{e}-06$ & $9.9772 \mathrm{e}-07$ \\
\hline$\theta \phi^{2}$ & $1.4263 \mathrm{e}-02$ & $-6.0037 e-02$ & $9.3111 \mathrm{e}-05$ & $-2.9056 e-04$ \\
\hline$\theta \phi \delta$ & $-1.4956 \mathrm{e}-04$ & $-9.0321 \mathrm{e}-04$ & $-1.2997 \mathrm{e}-06$ & $-4.5482 \mathrm{e}-06$ \\
\hline$\theta \delta^{2}$ & $2.7921 \mathrm{e}-10$ & $1.1758 \mathrm{e}-09$ & & \\
\hline$\phi^{3}$ & $-1.2762 \mathrm{e}-02$ & $-4.1164 \mathrm{e}-02$ & $-1.0749 \mathrm{e}-04$ & $-1.9591 \mathrm{e}-04$ \\
\hline$\phi^{2} \delta$ & $1.7196 \mathrm{e}-04$ & $7.3151 \mathrm{e}-04$ & $1.4890 \mathrm{e}-06$ & $3.5879 \mathrm{e}-06$ \\
\hline$\phi \delta^{2}$ & & $3.9880 \mathrm{e}-10$ & & \\
\hline$\theta^{3} \phi$ & $-5.3455 e-01$ & $-1.0787 \mathrm{e}+00$ & $-3.1460 \mathrm{e}-03$ & $-3.5412 \mathrm{e}-03$ \\
\hline$\theta^{3} \delta$ & $-3.2005 e-08$ & $2.2390 \mathrm{e}-07$ & $2.1175 \mathrm{e}-09$ & $9.1690 \mathrm{e}-10$ \\
\hline$\theta^{2} \phi^{2}$ & $4.3547 \mathrm{e}-01$ & $3.2656 \mathrm{e}-01$ & $5.6070 \mathrm{e}-03$ & $3.5352 \mathrm{e}-04$ \\
\hline$\theta^{2} \phi \delta$ & $-2.5480 \mathrm{e}-07$ & $-1.5288 \mathrm{e}-06$ & $-1.9612 \mathrm{e}-09$ & $-7.3195 e-09$ \\
\hline$\theta^{2} \delta^{2}$ & $-1.3981 \mathrm{e}-09$ & $-1.8994 \mathrm{e}-09$ & & \\
\hline$\theta \phi^{3}$ & $-9.9055 e-01$ & $-4.2915 e+00$ & $-5.3589 e-03$ & $-1.9464 \mathrm{e}-02$ \\
\hline$\theta \phi^{2} \delta$ & $9.8987 \mathrm{e}-08$ & $2.1943 \mathrm{e}-07$ & $1.8292 \mathrm{e}-09$ & $8.1178 \mathrm{e}-10$ \\
\hline$\theta \phi \delta^{2}$ & $-2.9432 \mathrm{e}-09$ & $-7.9973 \mathrm{e}-09$ & & \\
\hline$\phi^{3} \delta$ & $-5.9053 e-08$ & $-1.6683 e-06$ & $-4.3341 e-10$ & $-8.2847 \mathrm{e}-09$ \\
\hline$\phi^{2} \delta^{2}$ & $-1.8211 \mathrm{e}-09$ & $-1.1284 \mathrm{e}-08$ & & $-5.4851 \mathrm{e}-11$ \\
\hline & Variable & Order & Range & Mean \\
\hline & $\theta$ & 3 & 0.1 & 0 \\
\hline & $\phi$ & 3 & 0.1 & 0 \\
\hline & $\delta$ & 2 & 10 & $2.5 e+06$ \\
\hline
\end{tabular}

Table 3: Matrix of aberration coefficients for comparison of beamline in stray DC field with translation and translation-tilt alignment. 
strength of the lens, and thus is a negligible concern for microbeams.

An increase in chromatic aberration arises through to slight variation in the path taken through the stray DC magnetic field due to slight changes in particle momentum. This leads to slight misalignments of the optical elements to the optical axis of each particle/momentum, introducing steering and broadening aberration terms. The method used for evaluating these terms is straightforward. A polynomial equation is fitted to the output coordinates of rays resulting from a 3 dimensional matrix of input coordinates varied by divergence in the $\mathrm{X}-\mathrm{Z}$ plane, $\theta$, divergence in the $\mathrm{Y}-\mathrm{Z}$ plane, $\phi$, and small changes in the particle energy, delta. This process is repeated with the stray field removed from the simulation and elements re-aligned to the geometric axis, and the polynomial equations are compared.

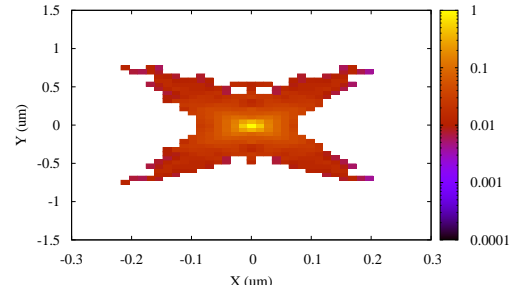

(a) Un-strayed

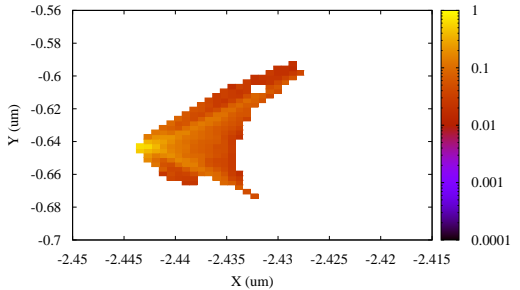

(c) Strayed / Unstrayed difference

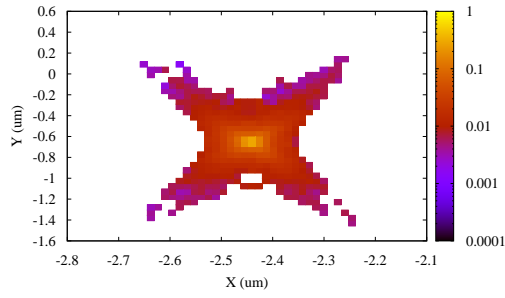

(b) Strayed focussed

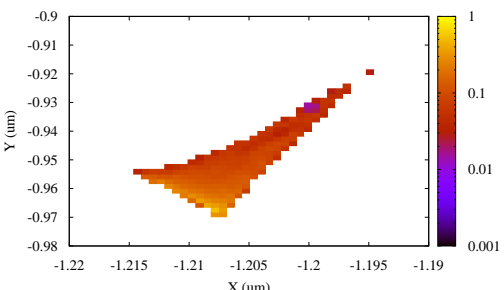

(d) Tilt aligned

Figure 1: Point spread function spots at image plane for alignment procedures to stray DC field, with chromatic aberration

The terminal voltage ripple in $2 \mathrm{MV}$ tandetron accelerator at the University of Surrey Ion Beam Centre has been shown to be less than $10^{-5}$ of the terminal voltage [3]. 
The increase in chromatic aberration due to the Earth's magnetic field shown in table ?? is negligible for this level of terminal voltage stability.

\subsection{Focussing performance degradation when changing beam energy}

In practice, when changing beam energy the microbeam operator will experimentally optimise beam current at the new beam energy by using any steering elements available before the object aperture such that the beam is matched to the acceptance defined by object and collimator apertures. This nullifies the influence of the stray field before the collimating aperture, however the pre-object alignment can only correct the tranverse offset of the beam to the beam geometric axis such that it arrives at the correct location in the collimating plane, whereas the stray field will cause the beam to travel in a curved path, causing a angular deviation from the geometric axis leaving the collimating aperture.

As such, the direction and radius of curvature of the beam leaving the collimating plane differs with magnetic rigidity of the beam, causing misalignment of postcollimator objects. In two-stage systems this may cause considerable misalignment due to the "early" position of the collimating aperture in the first stage - causing a large distance from collimating aperture to image plane.

It is necessary to fit the coefficient matrix to the 4 th order for the magnetic rigidity term such that the third order term $\chi^{3}$ can be fitted accurately. It is clear that there is a third order aberration associated with path changes due to variation of magnetic rigidity, as would be expected from high third order angular response of the lens system.

Displacement effects may be solved relatively easily by the use of fiducial marks on the sample holder, to provide accurate positions (requiring a recalibration for each change of rigidity). Beam broadening effects are more severe, and can only be corrected without re-alignment by reduction of the angular divergence of the beam.

The corresponding loss in beam current can be calculated and is shown in table TABLE for various rigidities. The reduction in beam current is calculated by reducing the angular divergence of the beam such that the contribution to the beam spot at the image is the same as that for the aligned rigidity. OR

It is possible to fit an aberration terms relating to the change in beam rigidity, the 
spot displacement and broadening, but the process is non-simple, since adjustment of the excitation of the optical elements is required for each change in energy.

When defining the beamline parameters for a simulation using XART it is possible to define function links in a similar fashion to "common blocks" in TRAX. The intention of this feature is to allow the coupling of power supplies in for example the first two quadrupoles of an Oxford Triplet, which require equal and opposite excitation. However, these links can be adapter to couple the momentum of the ion to the quadrupole excitation and alignment. A link can contain several functions, each with an independant ratio to the value of the link.

Therefore, there are four such links present in this simulation:

1. A link between excitation of first and second quadrupole lens.

2. A link between excitation of third lens, and first link.

3. A link between beam magnetic rigidity, second and third link, and the pre-object angular path of the beam.

This allows changes to be made to the final link, representing beam momentum, and the appropriate beamline alignment and excitation corrections are applied as the values are cascaded down through the remaining links. The core aberration fitting code of XART can then fit an aberration coefficient polynomial to the results of raytracing ions from a 3 dimensional matrix of input coordinates varied by divergence in both planes and beam momentum applied through the final link.

Since magnetic deflection is momentum dependant, rather than energy dependant, the aberrations in table 4 have been fitted to the magnetic rigidity of the beam, with units in tesla meters. Table 4 shows that strong first and second order aberrations terms produce an image displacement in the order of microns. The strength of aberration terms $\theta^{2} \chi, \phi^{2} \chi, \theta \chi^{2}, \phi \chi^{2}$, cause a strong parasitic broadening effect on the focussed image.

This is shown to be a major problem, not just in first order misalignment of the beam image, but also broadening terms exist. It is significant because the technque of proton beam writing may require greatly different ion energies to create multidepth structures. Igbenehi et al suggest a possible solution to this solution by using ions 
of different species but similar magnetic rigidities, therefore particles take exactly the same path through the stray fields. Insert reference here - ask Vladimir.

Table *** shows the magnitude of broadening and displacement effects due to the stray DC field for a range of ion rigidities for the Oxford Triplet beamline aligned for a beam of $2.5 \mathrm{MeV}$ protons.

Figure 2 shows the difference in point spread function when the magnetic rigidity of the beam is changed from $0.227 \mathrm{Tm}(2.5 \mathrm{MeV} \mathrm{H}+)$ to $0.203 \mathrm{Tm}(2.0 \mathrm{MeV} \mathrm{H}+)$ for an angular divergence in bot planes of $0.1 \mathrm{mrad}$.

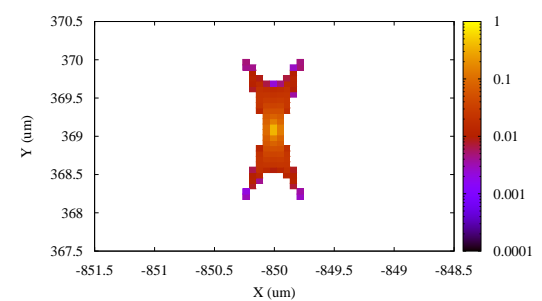

(a) $2.5 \mathrm{MeV}$ Aligned

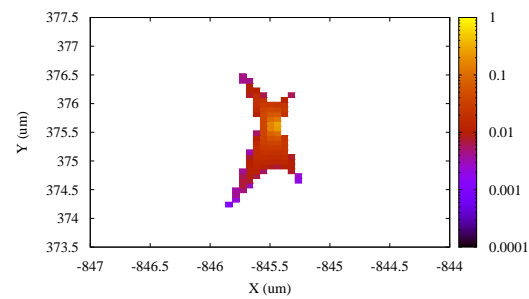

(b) $2.0 \mathrm{MeV}$ Misaligned

Figure 2: Point spread function spots at image plane when beamline is refocussed but not re-aligned.

\section{Conclusion}

It is clear that stray DC fields in a microbeam environment do produce intrinsic and chromatic beam aberrations, but that these are relatively small in magnitude in comparison to spherical aberration when optical elements are correctly aligned to the true path of the beam.

A more concerning effect is focus degradation

Future considerations may involve cancellation of stray DC magnetic fields in the beamline perhaps using helmholtz coils, however, this is non-simple and therefore has not been considered in this paper.

How does this affect the grid-shadow image? Considering that beam phase space may no longer be uniform since beam undergoes deflection in beamline. Also what 
Aberration matrix: units: $\mu \mathrm{m} / \operatorname{mrads} / \mathrm{Tm}$

\begin{tabular}{|l|cccc|}
\hline & $\mathrm{x}$ & $\mathrm{y}$ & $\theta$ & $\phi$ \\
\hline & $-8.5079 \mathrm{e}+02$ & $3.7073 \mathrm{e}+02$ & $-7.6467 \mathrm{e}-01$ & $3.4646 \mathrm{e}-01$ \\
$\theta$ & $7.8381 \mathrm{e}+00$ & $-1.6432 \mathrm{e}+01$ & $8.2485 \mathrm{e}+01$ & $-7.9725 \mathrm{e}-02$ \\
$\phi$ & $7.8891 \mathrm{e}+00$ & $-1.5470 \mathrm{e}+01$ & $8.9402 \mathrm{e}-02$ & $-3.1528 \mathrm{e}+01$ \\
$\chi$ & $-2.4946 \mathrm{e}+02$ & $-6.5156 \mathrm{e}+01$ & $2.9916 \mathrm{e}+01$ & $5.8045 \mathrm{e}+00$ \\
$\theta^{2}$ & $7.0935 \mathrm{e}+02$ & $-1.4736 \mathrm{e}+03$ & $8.1016 \mathrm{e}+00$ & $-7.1299 \mathrm{e}+00$ \\
$\theta \phi$ & $-8.1694 \mathrm{e}+01$ & $1.6430 \mathrm{e}+02$ & $-9.1273 \mathrm{e}-01$ & $7.9635 \mathrm{e}-01$ \\
$\theta \chi$ & $7.9079 \mathrm{e}+02$ & $-1.7883 \mathrm{e}+03$ & $9.5270 \mathrm{e}+00$ & $-8.5758 \mathrm{e}+00$ \\
$\phi^{2}$ & $7.0945 \mathrm{e}+02$ & $-1.4725 \mathrm{e}+03$ & $8.1022 \mathrm{e}+00$ & $-7.1229 \mathrm{e}+00$ \\
$\phi \chi$ & $7.8977 \mathrm{e}+02$ & $-1.7915 \mathrm{e}+03$ & $9.5278 \mathrm{e}+00$ & $-8.5867 \mathrm{e}+00$ \\
$\chi^{2}$ & $9.3228 \mathrm{e}+03$ & $-1.7282 \mathrm{e}+04$ & $-3.2464 \mathrm{e}+01$ & $-1.0957 \mathrm{e}+02$ \\
& & & & \\
$\theta^{3}$ & $-6.3695 \mathrm{e}+03$ & $1.4774 \mathrm{e}+04$ & $-7.6190 \mathrm{e}+01$ & $7.1439 \mathrm{e}+01$ \\
$\theta^{2} \phi$ & $-7.0947 \mathrm{e}+03$ & $1.0817 \mathrm{e}+04$ & $-8.1021 \mathrm{e}+01$ & $5.1537 \mathrm{e}+01$ \\
$\theta^{2} \chi$ & $7.2005 \mathrm{e}+04$ & $-1.6038 \mathrm{e}+05$ & $8.6322 \mathrm{e}+02$ & $-7.6868 \mathrm{e}+02$ \\
$\theta \phi^{2}$ & $-5.5838 \mathrm{e}+03$ & $1.4774 \mathrm{e}+04$ & $-6.8023 \mathrm{e}+01$ & $7.1438 \mathrm{e}+01$ \\
$\theta \phi \chi$ & $-8.5025 \mathrm{e}+03$ & $1.4803 \mathrm{e}+04$ & $-1.0038 \mathrm{e}+02$ & $7.0262 \mathrm{e}+01$ \\
$\theta \chi^{2}$ & $-8.5369 \mathrm{e}+04$ & $1.8414 \mathrm{e}+05$ & $-1.0264 \mathrm{e}+03$ & $8.8186 \mathrm{e}+02$ \\
$\phi^{3}$ & $-7.0947 \mathrm{e}+03$ & $9.9712 \mathrm{e}+03$ & $-8.1021 \mathrm{e}+01$ & $4.7905 \mathrm{e}+01$ \\
$\phi^{2} \chi$ & $7.1754 \mathrm{e}+04$ & $-1.5833 \mathrm{e}+05$ & $8.6292 \mathrm{e}+02$ & $-7.5873 \mathrm{e}+02$ \\
$\phi \chi^{2}$ & $-8.5982 \mathrm{e}+04$ & $1.8237 \mathrm{e}+05$ & $-1.0313 \mathrm{e}+03$ & $8.7306 \mathrm{e}+02$ \\
\hline \hline & Variable & Order & Range & Mean \\
\cline { 2 - 5 } & $\theta$ & 3 & 0.1 & 0 \\
& $\phi$ & 3 & 0.1 & 0 \\
& $\chi$ & 2 & 0.01 & 0.228014 \\
\hline
\end{tabular}

Table 4: Matrix of aberration coefficients for magnetic rigidity aberration in Earths magnetic field.

\begin{tabular}{|c|c|c|c|c|c|c|}
\hline Mass (amu) & $1.000 \mathrm{E}+0$ & $1.000 \mathrm{E}+0$ & $1.000 \mathrm{E}+0$ & $1.000 \mathrm{E}+0$ & $1.000 \mathrm{E}+0$ & $4.000 \mathrm{E}+0$ \\
\hline Charge $(\mathrm{eV})$ & $1.000 \mathrm{E}+0$ & $1.000 \mathrm{E}+0$ & $1.000 \mathrm{E}+0$ & $1.000 \mathrm{E}+0$ & $1.000 \mathrm{E}+0$ & $2.000 \mathrm{E}+0$ \\
\hline Rigidity difference (Tm) & $-8.367 \mathrm{E}-2$ & $-5.131 \mathrm{E}-2$ & $-2.403 \mathrm{E}-2$ & $0.000 \mathrm{E}+0$ & $2.173 \mathrm{E}-2$ & $9.429 \mathrm{E}-2$ \\
\hline Rigidity $(\mathrm{Tm})$ & $1.440 \mathrm{E}-1$ & $1.763 \mathrm{E}-1$ & $2.036 \mathrm{E}-1$ & $2.276 \mathrm{E}-1$ & $2.494 \mathrm{E}-1$ & $3.219 \mathrm{E}-1$ \\
\hline Energy $(\mathrm{MeV})$ & $1.000 \mathrm{E}+0$ & $1.500 \mathrm{E}+0$ & $2.000 \mathrm{E}+0$ & $2.500 \mathrm{E}+0$ & $3.000 \mathrm{E}+0$ & $2.500 \mathrm{E}+0$ \\
\hline Velocity $\left.\left(m \cdot s^{-1}\right)\right)$ & $1.389 \mathrm{E}+7$ & $1.701 \mathrm{E}+7$ & $1.965 \mathrm{E}+7$ & $2.196 \mathrm{E}+7$ & $2.406 \mathrm{E}+7$ & $1.553 \mathrm{E}+7$ \\
\hline Image Displacement X $(\mu \mathrm{m})$ & $2.202 \mathrm{E}+1$ & $1.122 \mathrm{E}+1$ & $4.561 \mathrm{E}+0$ & $0.000 \mathrm{E}+0$ & $-3.234 \mathrm{E}+0$ & $-1.104 \mathrm{E}+1$ \\
\hline Image Displacement $Y(\mu m)$ & $3.148 \mathrm{E}+1$ & $1.607 \mathrm{E}+1$ & $6.536 \mathrm{E}+0$ & $0.000 \mathrm{E}+0$ & $-4.834 \mathrm{E}+0$ & $-1.583 \mathrm{E}+1$ \\
\hline Broadening $X(\mu m)$ & $2.259 \mathrm{E}+1$ & $3.435 \mathrm{E}+0$ & $1.500 \mathrm{E}-1$ & $0.000 \mathrm{E}+0$ & $4.347 \mathrm{E}-2$ & $2.351 \mathrm{E}+1$ \\
\hline Broadening Y $(\mu m)$ & $-4.686 \mathrm{E}+1$ & $-7.267 \mathrm{E}+0$ & $-3.967 \mathrm{E}-1$ & $0.000 \mathrm{E}+0$ & $-1.722 \mathrm{E}-2$ & $-4.813 \mathrm{E}+1$ \\
\hline Beam Current Loss & & & & & & \\
\hline
\end{tabular}

Table 5: Displacement and Broadening of the image due to change in magnetic rigidity of ion from alignment rigidity $(0.228 \mathrm{Tm})$. 


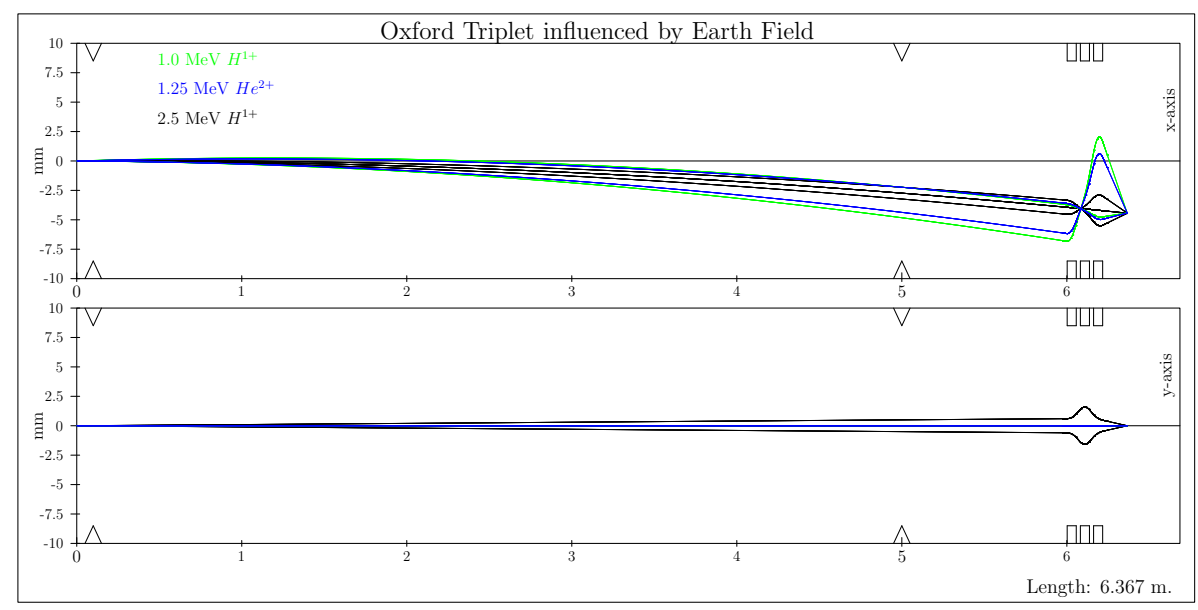

Figure 3: Raytracing simulations of multiple rigidities in Oxford Triplet

will happen if the beam is still influenced by the stray field in the region between the grid and the shadow?

Different beamline geometries will respond differently to changes in rigidity with a simple doublet likely to be least susceptible due to it's low intrinsic aberration, and low number of lenses. However the performance of such a system is far less than that of high performance systems, and the beamline designer must find a balance between performance and susceptibility to stray fields, amongst many other parasitic effects degrading beamline performance.

It may be possible to adapt this method such that an aberration term relating to the strength of the stray field can be calculated, however, the influence of the stray field depends on a large number of parameters; orientation, location, strength, as such it is not desirable to perform this calculation, instead calculating the broadening effect due to a specific field.

This effect may be important to the proton beam writing community who commonly use several different ion energies.

These results may be particularly painful to monoblock lens assemblies where lenses cannot be either translated or tilted individually.

At higher rigidities particles will receive less deflection from the Earth's magnetic field, and thus aberrations introduced from lens misalignment will be reduced. 


\section{References}

[1] G.W. Grime and F. Watt. Beam Optics of Quadrupole Probe Forming Systems. Adam Hilger Ltd, Bristol, 1984.

[2] The National Geophysical Data Center (NGDC). Geomagnetism calculator. http://www.ngdc.noaa.gov/geomag/magfield.shtml, November 2008.

[3] A. Simon, C. Jeynes, R.P. Webb, R. Finnis, Z. Tabatabaian, P.J. Sellin, M.B.H. Breese, D.F. Fellows, R. van den Broek, and R.M. Gwilliam. The new surrey ion beam analysis facility. Nuclear Instruments and Methods in Physics Research Section B, 219-220:405-409, 2004.

[4] F. Watt, G.W. Grime, G.D. Blower, J. Takacs, and D.J.T. Vaux. The oxford one micron proton microprobe. Nuclear Instruments and Methods in Physics Research, 197:65, 1982. 